\title{
Downregulation of YAP inhibits proliferation, invasion and increases cisplatin sensitivity in human hepatocellular carcinoma cells
}

\author{
XIAOGUANG WANG, BIN WU and ZHENGXIANG ZHONG \\ Department of Hepatobiliary Surgery, The Second Affiliated Hospital of Jiaxing Medical College, \\ Jiaxing, Zhejiang 314000, P.R. China
}

Received August 31, 2017; Accepted March 1, 2018

DOI: $10.3892 / \mathrm{ol} .2018 .8633$

\begin{abstract}
Yes-associated protein (YAP) serves an essential role in tumorigenesis. However, the potential role and the molecular mechanism underlying the effect of YAP on hepatocellular carcinoma (HCC) cells have not been elucidated. In the current study, it was revealed that YAP expression was increased significantly in HCC cancer tissues and its overexpression was associated with tumor differentiation. The silencing of YAP by small interferring RNA led to the inhibition of HCC cell growth, which was associated with the promotion of apoptosis. The silencing of YAP also decreased the invasive potential of HCC cells and the activity of the phosphoinositide 3-kinase (PI3K)/AKT serine/threonine kinase (AKT) signaling pathway. Furthermore, silencing of YAP increased the chemosensitivity of HCC cells to cisplatin (CDDP) through inactivation of the PI3K/AKT signaling pathway. In vivo studies using PDTX model suggested a promotive role for YAP in the growth of HCC and knockdown of YAP increased the anti-tumor activity of CDDP. Taken together, these results revealed that YAP is overexpressed in $\mathrm{HCC}$, and promotes proliferation, invasion and drug resistance of $\mathrm{HCC}$ cells. Inhibition of YAP, alone or in combination with traditional chemotherapy, may effectively combat HCC.
\end{abstract}

\section{Introduction}

Hepatocellular carcinoma (HCC) is the third most common cause of cancer-associated mortality worldwide (1). There are $>250,000$ new HCC cases and an estimated 600,000 HCC

Correspondence to: Dr Zhengxiang Zhong, Department of Hepatobiliary Surgery, The Second Affiliated Hospital of Jiaxing Medical College, Building 9, 1518 HuanCheng Road, Jiaxing, Zhejiang 314000, P.R. China

E-mail: zay198699@163.com

Key words: yes-associated protein, cisplatin, chemoresistance, proliferation, invasion, hepatocellular carcinoma mortalities each year worldwide $(1,2)$. Although the combination of surgery and chemotherapy has increased the survival time of patients with HCC in recent years, a significant number of patients still relapse due to the resistance of tumor cells to chemotherapeutic agents $(3,4)$. Unfortunately, the mechanisms underlying HCC chemoresistance remain unclear. Therefore, the identification of the genes and proteins that regulate chemoresistance is essential for the exploration of novel targeted therapies for $\mathrm{HCC}$, which will be beneficial for a large group of patients.

Yes-associated protein (YAP), a key component of the Hippo signaling pathway, serves roles in development, growth, repair and homeostasis $(5,6)$. Accumulating evidence has implicated that YAP serves an important and multifaceted role in cancer progression, including gastric cancer, pediatric cancer and breast cancer (7-10). YAP also been demonstrated to be overexpressed in various types of solid tumors, including gastric cancer, pediatric cancer and breast cancer, and its overexpression is associated with tumor initiation, invasion and metastasis $(9,11,12)$. These characteristics make YAP an attractive therapeutic target in cancer treatment. In HCC, YAP has been revealed to be involved in proliferation, migration, invasion and drug resistance (13-15). However, the underlying mechanisms remain unclear.

In the present study, the effects of YAP expression manipulation on proliferation, invasion, cisplatin (CDDP) resistance were investigated, and its mechanism in HCC cells was explored. It was demonstrated that YAP overexpression is associated with the tumor differentiation in HCC. Downregulation of YAP using small interfering (si)RNA inhibited the proliferation and invasion of HCC cells, and resulted in a significant decrease in the activity of the phosphoinositide 3-kinase (PI3K)/AKT serine/threonine kinase (AKT) signaling pathway. Furthermore, the knockdown of YAP increased the sensitivity of HCC cells to CDDP via inhibition of the PI3K/AKT signaling pathway. In a patient-derived tumor xenograft (PDTX) model, the knockdown of YAP inhibited the growth of HCC, and also increased the anti-tumor activity of CDDP. Therefore, the findings of the present study suggest a promoter role for YAP on HCC. Inhibition of YAP, alone or in combination with traditional chemotherapy, may effectively combat HCC. 


\section{Materials and methods}

Patients and tissue samples. The present study was approved and supervised by the Research Ethics Committee of Jiaxing University College of Medicine (Jiaxing, China). Written informed consent was obtained from all patients. Paired liver cancer and adjacent normal liver tissue were obtained between September 2015 and December 2017 from 80 patients who underwent primary surgical resection of liver cancer at The Second Affiliated Hospital of Jiaxing University College of Medicine. The patients included 36 women and 44 men, aged 36-81 years; 32 patients had WHO grade T1 disease, 28 patients had WHO grade T2 disease, and 16 patients had WHO grade T3, 4 patients had WHO grade T4 disease (16). All tissue samples were immediately frozen in liquid nitrogen and stored at $-80^{\circ} \mathrm{C}$ until total RNA and protein was extracted.

Cell culture. SMMC-7721 cells (derived from liver tissues of a male patient with HCC) and THLE-3 cells (a normal liver cell line) were purchased from the Cell Bank of the Shanghai Institute of Biochemistry and Cell Biology (Shanghai, China), where the cell line was tested and authenticated. These procedures include cross-species checks, DNA authentication and quarantine. Cells were placed in culture for $<6$ months and authenticated at The Second Affiliated Hospital of Jiaxing Medical College using morphology and growth rate assays. SMMC-7721 cells were maintained in Dulbecco's modified Eagle medium (DMEM; Hyclone; GE Healthcare Life Sciences, Logan, UT, USA) containing 10\% fetal bovine serum (FBS; Gibco; Thermo Fisher Scientific, Inc., Waltham, MA, USA) and incubated at $37^{\circ} \mathrm{C}$ in a $5 \% \mathrm{CO}_{2}$ atmosphere. THLE-3 cells were cultured in BEGM medium kit (Lonza/Clonetics Corporation, Walkersville) and incubated at $37^{\circ} \mathrm{C}$ in a $5 \% \mathrm{CO}_{2}$ atmosphere.

Immunohistochemistry. For immunohistochemistry, paraffin-embedded sections of 5- $\mu \mathrm{m}$ thickness were deparaffinised in $100 \%$ xylene for $20 \mathrm{~min}$ at room temperature, and rehydrated in graded ethyl alcohol (100, 90, 70 and 50\% ethyl alcohol) for $10 \mathrm{~min}$ at room temperature. Endogenous peroxidase activity was blocked with 3\% hydrogen peroxide in 100\% methanol for $10 \mathrm{~min}$ at room temperature. Antigen retrieval was performed in sodium citrate buffer for $2 \mathrm{~h}$ at room temperature. The rabbit polyclonal antibody directed against YAP (cat. no. ab39361; 1:100 dilution; Abcam, Cambridge, MA, USA) was added to sections and incubated at $4^{\circ} \mathrm{C}$ overnight. A horseradish peroxidase (HRP)-conjugated anti-rabbit $\operatorname{IgG}$ (cat. no. ab6721, 1:1,000 dilution, Abcam) was used according to the manufacturer's protocol. The slides were then incubated with DAB (Beyotime Institute of Biotechnology, Haimen, China) for $5 \mathrm{~min}$ at room temperature, and then YAP expression was visualised, followed by $0.5 \%$ hematoxylin (Beyotime Institute of Biotechnology) counterstaining for $10 \mathrm{~min}$ at room temperature. PBS was used as a negative control. The images were captured using a camera connected to an Olympus BH2 light microscope (Olympus Corporation, Tokyo, Japan) at magnifications, $x 100$ and $\times 200$. Staining results were assessed independently by two pathologists blinded to the clinical data of patients. The intensity of YAP protein staining for each slide was scored and accessed according to the criterion based on the intensity of staining: 0 , negative; $1+$, low; $2+$, medium;
$3+$, high. The percentage of stained cells was calculated as: $0,0 \%$ stained; $1+, 1-10 \%$ stained; $2+, 11-49 \%$ stained; $3+$, $50-100 \%$ stained. YAP IHC data were analyzed using X-tile 1.9 software program (The Rimm Lab at Yale University, New Haven, CT, USA; http://www.tissuearray.org/rimmlab).

Reverse transcription-quantitative polymerase chain reaction. Total RNA was extracted from cells or tumor samples using TRIzol ${ }^{\circledR}$ (Invitrogen; Thermo Fisher Scientific, Inc.), and $2 \mu \mathrm{g}$ of total RNA from each sample was used for cDNA synthesis using a cDNA synthesis kit (Tiangen Biotech Co., Ltd., Beijing, China). The qPCR analysis was performed using PowerUp ${ }^{\mathrm{TM}}$ SYBR $^{\circledR}$ Green Master Mix (cat. no. A25741; Thermo Fisher Scientific, Inc.) containing Dual-Lock Taq DNA Polymerase, according to the manufacturer's protocol. Data collection was conducted using an ABI 7500 (Applied Biosystems; Thermo Fisher Scientific, Inc.). The thermocycling conditions were as follows: $50^{\circ} \mathrm{C}$ for $2 \mathrm{~min}$ and $95^{\circ} \mathrm{C}$ for $2 \mathrm{~min}$, followed by 40 cycles of $95^{\circ} \mathrm{C}$ for $15 \mathrm{sec}$ and $60^{\circ} \mathrm{C}$ for $1 \mathrm{~min}$. The conditions for melt curve analysis were $95^{\circ} \mathrm{C}$ for $15 \mathrm{sec}, 60^{\circ} \mathrm{C}$ for $1 \mathrm{~min}$ and $95^{\circ} \mathrm{C}$ for $15 \mathrm{~min}$. PCR amplification was conducted using the following primers: YAP forward, 5'-CCCTCGTTT TGCCATGAACC-3' and reverse, 5'-ATCTGTTGCTGCTGG TTGGA-3'; $\beta$-actin forward, 5'-GATGAGATTGGCATG GCTTT-3' and reverse, 5'-GTCACCTTCACCGTTCCAGT-3'. The $2^{-\Delta \Delta C a}$ method (17) was used to calculate gene expression levels. The level of each RNA sample was normalized to that of the housekeeping gene $\beta$-actin.

Western blot analysis. Whole-cell extracts from cultured cells or tissues were prepared with radioimmunoprecipitation assay buffer (Beyotime Institute of Biotechnology) and subjected to western blotting. Protein concentration was analyzed using the Pierce ${ }^{\mathrm{TM}}$ BCA Protein Assay kit (cat. no. 2322; Invitrogen; Thermo Fisher Scientific, Inc.). Total protein (40 $\mu \mathrm{g} / \mathrm{lane})$ was separated by $12 \%$ SDS-PAGE and transferred into polyvinylidene fluoride membranse (EMD Millipore, Billerica, MA, USA). The membranes were then blocked with 5\% skimmed milk powder for $1 \mathrm{~h}$ at room temperature. The membranes were then incubated with primary antibodies overnight at $4^{\circ} \mathrm{C}$. All primary antibodies were purchased from Abcam (Cambridge, UK) as follows: Rabbit anti-YAP1 antibody (cat. no. ab39361; dilution, 1:1,000), mouse anti- $\beta$-actin antibody (cat. no. ab8226; dilution, 1:10,000), rabbit anti-pan-AKT antibody (cat. no. ab8805; dilution, 1:500), Rb anti-pan-AKT (phospho T308) antibody (cat. no. ab38449; dilution, 1:800), rabbit anti-p21 (cat. no. ab109199; dilution, 1:5,000), rabbit anti-Bax (cat. no. ab32503; dilution, 1:1,000), rabbit anti-active-Caspase-3 (cat. no. ab2302; dilution, 1:200) and rabbit anti-c-myc (cat. no. ab32072, dilution, 1:10,000). The membranes were then washed three times with PBS-Tween-20 for $10 \mathrm{~min}$ and then incubated with the HRP-conjugated goat anti-mouse IgG H\&L (cat. no. ab6789; dilution, 1:5,000; Abcam) or goat anti-rabbit IgG H\& L (HRP) (cat. no. ab6721; dilution, 1:10,000; Abcam) secondary antibodies for $1 \mathrm{~h}$ at room temperature. The blots were developed using an enhanced chemiluminescence Western Blotting Detection system (Thermo Fisher Scientific, Inc.) and X-ray film. The band density was quantified using Image J v1.48 u software (National Institutes of Health, Bethesda, MD, USA). 
Transfection and treatments. pcDNA3.1 vector containing wild type YAP insert was provided by General Biosystems, Inc. (Morrisville, NC, USA). siRNA targeted against YAP (Shanghai GenePharma Co., Ltd., Shanghai, China) with the sequence, 5'-GGUGAUACUAUCAACCAAAdTdT-3' was used to knock down YAP expression and the sequence of negative control siRNA was 5'-CAGUACUUUUGUGUAGUA CAAdTdT-3'. Cells ( $5 \times 10^{5}$ cells/well) were cultured in 6-well plates until $60 \%$ confluent, and then transfected with plasmids (4 $\mu \mathrm{g} /$ well) or siRNAs (20 $\mathrm{nM} /$ well) using Lipofectamine $2000^{\circledR}$ (Invitrogen; Thermo Fisher Scientific, Inc.) according to the manufacturer's protocol. LY294002 (Selleck Chemicals, Houston, TX, USA), dissolved in $1 \%$ DMSO, was added $24 \mathrm{~h}$ following transfection and used at $10 \mu \mathrm{M}$.

Cell invasion assay. The invasion chambers with 0.8-mm pore size (BD Biosciences, Franklin Lakes, NJ, USA) was coated with Matrigel (cat. no. 354234; 1:3 dilution; BD Biosciences) at $37^{\circ} \mathrm{C}$ for $30 \mathrm{~min}$. . A total of $\sim 1 \times 10^{5}$ cells suspended in DMEM medium with $2 \%$ FBS were added to the upper chamber, and the medium containing $20 \% \mathrm{FBS}$ was added to the lower chamber. After $24 \mathrm{~h}$ of incubation at $37^{\circ} \mathrm{C}$ in $5 \% \mathrm{CO}_{2}$, cells were fixed with $100 \%$ ice-cold methanol for $30 \mathrm{~min}$ at room temperature, and stained with $5 \%$ crystal violet for $15 \mathrm{~min}$ at room temperature. Then, cells on the upper surface of the inner chamber were removed with cotton swabs. Invaded cells that adhered to the lower surface of the membrane were viewed through an optical microscope (TS100F, Nikon, Japan) at magnification, $\mathrm{x} 100$, and counted using Image $\mathrm{J}$ v1.48u software.

MTT assay. An MTT assay was performed to measure cell viability. Briefly, cells $\left(5 \times 10^{3}\right.$ cells/well) were cultured in 96-well plates for $24 \mathrm{~h}$, transfected with YAP siRNA or YAP expression plasmids, and cell viability was determined $48 \mathrm{~h}$ later. The siRNA-trasfected HCC cells were exposed to CDDP (cat. no. S1166, Selleck Chemicals, Houston, TX, USA) at 0, 1, $10,20,40$ or $80 \mu \mathrm{M}$ for $48 \mathrm{~h}$ or treated with $10 \mu \mathrm{M}$ LY294002 for $12 \mathrm{~h}$ prior to being treated with CDDP. To determine the cell viability, MTT solution (5 $\mathrm{mg} / \mathrm{ml}$ in PBS) was added to each well and incubated at $37^{\circ} \mathrm{C}$ for $4 \mathrm{~h}$. The media was removed and $150 \mu \mathrm{l}$ dimethyl sulfoxide was added to each well. The absorbance of each well was measured at $490 \mathrm{~nm}$ using an automated microplate reader.

Cell apoptosis assay. Cell apoptosis was evaluated by flow cytometry using an Annexin-V-fluorescein isothiocyanate (FITC) Apoptosis Detection kit (BD Biosciences) according to the manufacturer's protocol. Briefly, the cells were harvested at $160 \mathrm{x} \mathrm{g}$ for $5 \mathrm{~min}$ at room temperature and washed twice in PBS and resuspended in $500 \mu 1$ of binding buffer. A volume of $5 \mu \mathrm{l}$ of Annexin-V-FITC and $5 \mu \mathrm{l}$ of propidium iodide was added and agitated gently, and the cells were stained in the dark for $15 \mathrm{~min}$ at room temperature. The cells were analyzed immediately by flow cytometry and analyzed using FlowJo 10.0 (Tree Star., Inc., Ashland, OR, USA).

Detection of bromodeoxyuridine (BrdU) incorporation. Untreated cells, or cells transfected with plasmid or siRNA were washed thoroughly with medium and cultured in fresh DMEM containing 10\% FBS and $10 \mu \mathrm{M}$ BrdU (Sigma-Aldrich; Merck KGaA, Darmstadt, Germany) for $1 \mathrm{~h}$ at $37^{\circ} \mathrm{C}$. Cells were then harvested at $160 \mathrm{x}$ g for $5 \mathrm{~min}$ at room temperature or left to grow in BrdU-free medium $24 \mathrm{~h}$ prior to harvest. Cell pellets were washed with PBS, fixed in $70 \%$ ice-cold ethanol for $20 \mathrm{~min}$ at room temperature, and then were resuspended in $2 \mathrm{~N} \mathrm{HCl}$ and incubated for $30 \mathrm{~min}$ at room temperature. Following washing with PBS, cells were hybridized with a mouse monoclonal anti-BrdU antibody (Abcam, cat. no. ab8152) diluted at a ratio 1:100 in PBST (PBS containing 0.1\% BSA and $0.2 \%$ Tween-20, pH 7.4) and incubated overnight at $4^{\circ} \mathrm{C}$. Cells were then washed with PBST and incubated with FITC-conjugated goat anti-mouse immunoglobulin antibody (cat. no. 715-545-155; Jackson Immuno Research Laboratories, Inc., West Grove, PA, USA) diluted at a ratio 1:400 in PBST for $2 \mathrm{~h}$ at room temperature in the dark. Cells were then washed with PBS and stained with DAPI solution for $10 \mathrm{~min}$ at room temperature prior to capturing images under a light microscope (BX43, Olympus, Japan).

Animal experiments. To produce the liver PDTX model, 6-8 week old male nu/nu mice (Laboratory Animal Center of Jiaxing University) weighing 18-20 g were used. All mice experiments were performed in accordance with the guidelines and approved by the Institutional Animal Care and Use Committee of Jiaxing University. Fresh surgical tumor tissues (F0) were collected immediately following surgery and cut into 2-3 $\mathrm{mm}^{3}$-sized pieces in Penicillin-Streptomycin Solution (Beyotime Institute of Biotechnology)-containing DMEM. Tumor fragments were implanted into the right armpit of mice. When the tumor size reached $100-200 \mathrm{~mm}^{3}$, the samples $(\mathrm{F} 1)$ were subsequently divided into pieces for passaging in vivo to make F2 xenograft tumors. When F2 tumors had reached a size $100-200 \mathrm{~mm}^{3}$, they were collected and cut into $2-3 \mathrm{~mm}^{3}$ sized pieces, then implanted into the right armpit of mice to make F3. When F3 tumor sizes had reached 100-200 $\mathrm{mm}^{3}$, mice were randomly divided into four groups with three mice/group.

The four groups were injected intravenously into the tail once a week with stroke-physiology saline solution, CDDP (5 mg/kg), YAP-shRNA lentivector (5 million infection units per $100 \mu \mathrm{l}$ for animal injection; Obio Technology Corp, Ltd., Shanghai, China) respectively or CDDP in combination with YAP-shRNA lentivector. Tumor diameters were serially measured with a digital caliper every 5 days, and tumor volumes were calculated using the following formula: $(\mathrm{L} \times \mathrm{W} \times \mathrm{W}) / 2$, whereby; V, volume; L, length; and $\mathrm{W}$, width. On day 25, mice were sacrificed and tumor tissues were collected.

Statistical analysis. Statistical analysis was performed using SPSS 17.0 software (IBM Corp., Armonk, NY, USA). Statistical analysis was performed using one-way analysis of variance followed Tukey's multiple comparisons test. Results are expressed as the mean \pm standard deiviation. $\mathrm{P}<0.05$ was considered to indicate a statistically significant difference.

\section{Results}

YAP is upregulated in human HCC tissues. To understand the role of YAP in HCC, the expression of YAP was examined in 


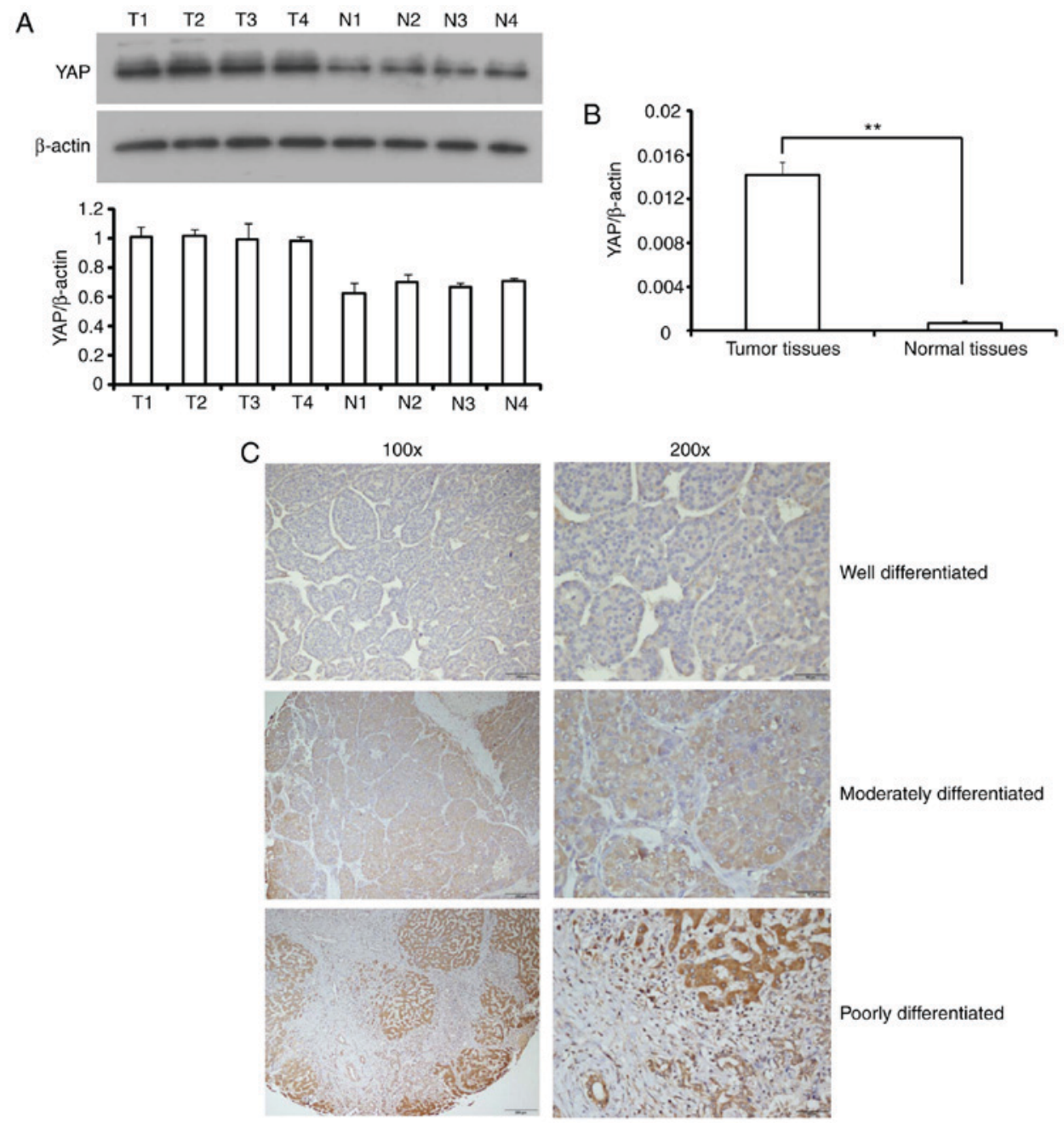

Figure 1. Expression of YAP is increased in HCC. (A) Representative western blot analysis of YAP protein in HCC (T1-T4) and paired normal tissues (N1-N4) from 4 patients. The expression of $\beta$-actin used as a loading control to normalize the YAP protein levels in each sample. (B) Determination of YAP mRNA level in HCC tissues and paired normal tissues by reverse transcription-quantitative polymerase chain reaction. ${ }^{* *} \mathrm{P}<0.01$. (C) Expression of YAP was analyzed by immunohistochemistry in HCC of different differentiation degrees using an anti-YAP antibody. Scale bars of the left-hand panels, $200 \mu \mathrm{m}$; scale bars of the right-hand panels, $100 \mu \mathrm{m}$. YAP, Yes-associated protein; HCC, hepatocellular carcinoma.

HCC tissues and adjacent normal tissues. The results of western blotting and RT-qPCR revealed that YAP expression in HCC tissues was significantly higher compared with peri-tumor tissues at the mRNA and protein levels (Fig. 1A and B). The expression of YAP was also analyzed using immunohistochemistry in paraffin-embedded HCC tissues. Representative images of immunohistochemical staining of tissues with YAP antibody demonstrated that the YAP-positive area increased markedly in poorly differentiated HCC compared with well differentiated HCC (Fig. 1C).

Downregulation of YAP inhibits the proliferation of HCC cells in vitro. In order to determine if YAP serves a functional role in HCC cell behavior in vitro, the level of YAP expression in the HCC cell line, SMMC-7721 was determined. SMMC-7721 cells had significantly highed basal YAP protein expression compared with the normal liver THLE-3 cell line (Fig. 2A). SMMC-7721 cells were then transfected with YAP siRNA or control siRNA. The YAP protein expression level was successfully reduced in SMMC-7721 cells by $78.5 \%$ compared with control siRNA-transfected cells (Fig. 2B), which was reversed following the transfection with YAP cDNA. As shown in Fig. 2C, transfection with YAP siRNA significatly reduced the viability of SMMC-7721 cells compared with control cells, which was restored by the overexpression of YAP in the knockdown cells. The BrdU assay revealed that YAP knockdown cells exhibited diminished proliferative capacity, which was rescued by re-expressing YAP (Fig. 2D). To determine whether the growth-inhibitory effects of YAP siRNA were due to increased cell death, cell apoptosis rates were determined by flow cytometry and the results demonstrated that YAP siRNA significantly induced apoptosis of SMMC-7721 cells after $48 \mathrm{~h}$ of transfection (Fig. 2E). Furthermore, the levels of several apoptosis-associated proteins were detected by western blot analysis. As shown in Fig. 2F, the protein levels of caspase 3, BCL2 associated X apoptosis regulator and p21 were significantly increased, whereas the expression level of c-myc was remarkably reduced in YAP siRNA-transfected SMMC-7721 cells compared with the control siRNA group. Collectively, these data suggested that the observed decrease in the number of cells upon YAP siRNA transfection was partially due to increased cell apoptosis. 

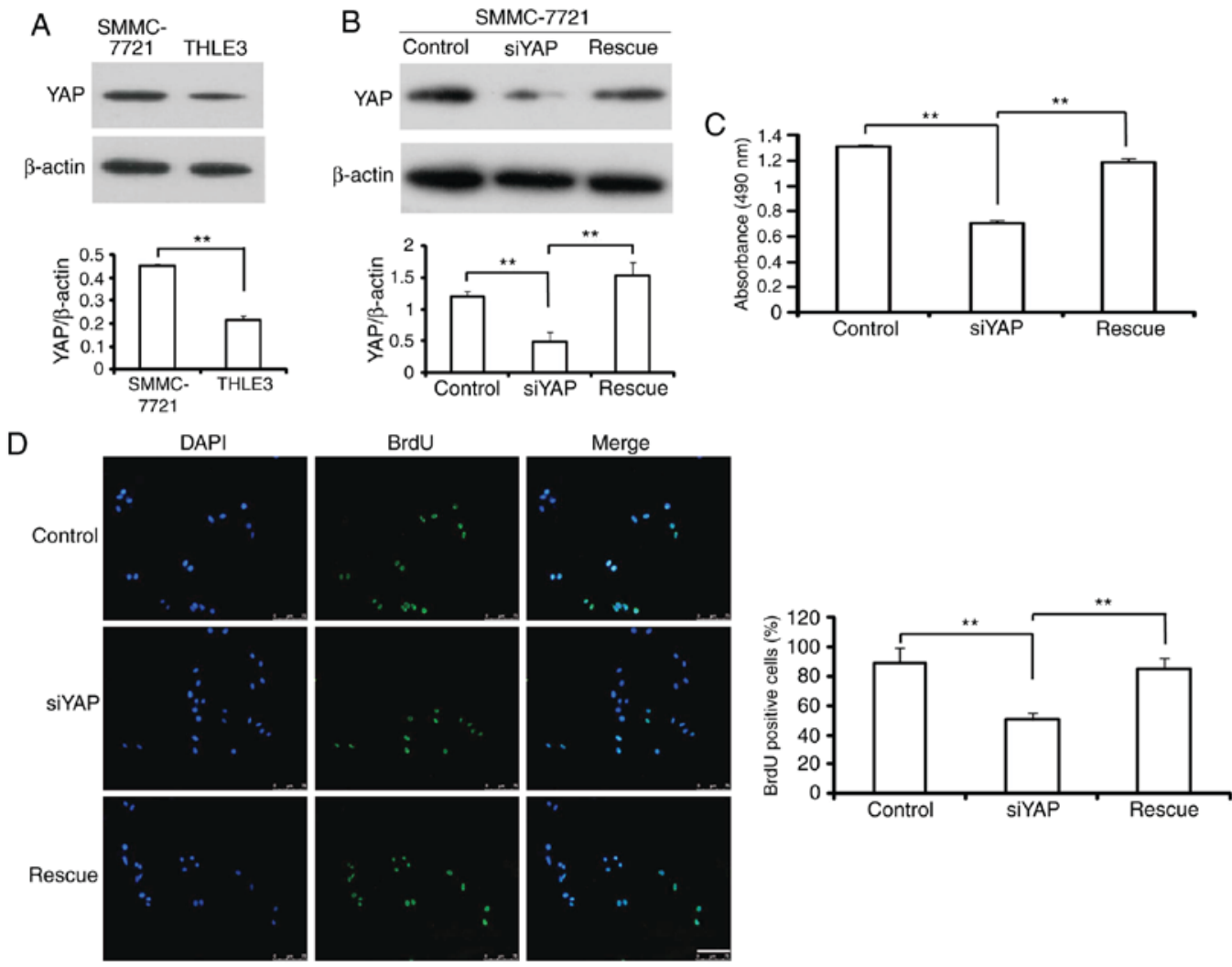

SMMC-7721
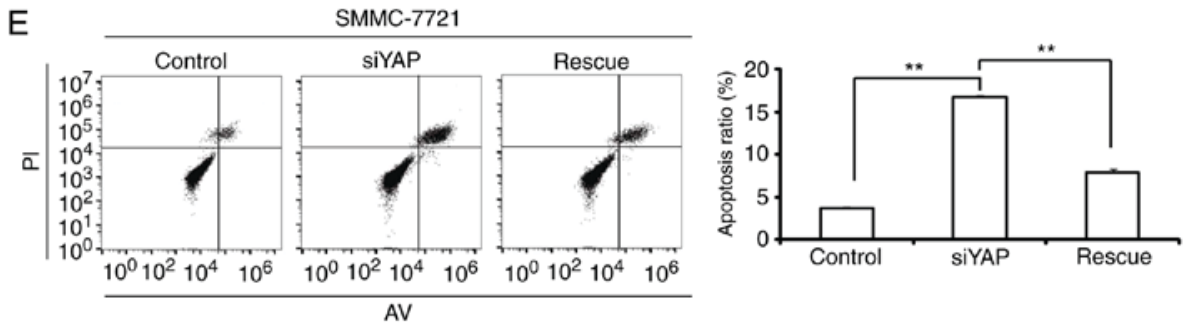

$\mathrm{F}$
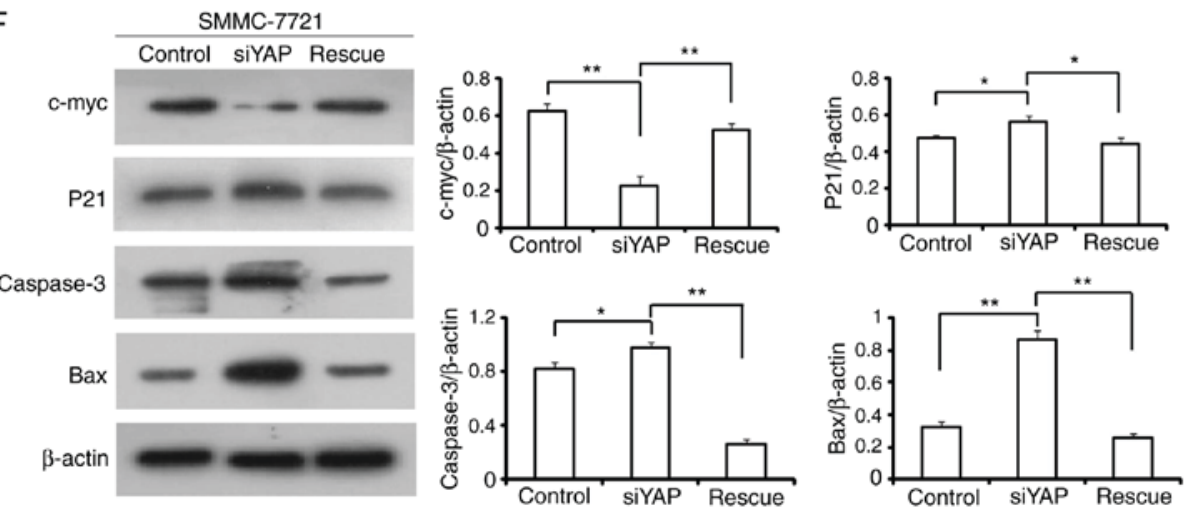

Figure 2. YAP knockdown inhibits hepatocellular carcinoma cell proliferation. (A) YAP expression is determined in a normal liver cell line and a HCC cell line by western blot analysis. (B) YAP expression in SMMC-7721 cells following transfection with control siRNA (control), YAP siRNA (siYAP) or YAP siRNA and YAP cDNA (Rescue) was measured by western blot analysis. $\beta$-actin served as a loading control. (C and D) The effect of YAP siRNA on cell growth. SMMC-7721 cells transiently transfected with control siRNA, YAP siRNA or YAP siRNA and YAP cDNA were cultured for $72 \mathrm{~h}$. (C) Cell viability was measured using an MTT assay and (D) cell proliferation was measured with a BrdU assay. Scale bar, $75 \mu \mathrm{m}$. (E) Flow cytometry analysis of SMMC-7721 cells. (F) Western blot analysis of c-myc, p21, caspase 3 and Bax protein expression levels in transfected cells. ${ }^{*} \mathrm{P}<0.05 ;{ }^{* *} \mathrm{P}<0.01$. YAP, Yes-associated protein; siRNA/si, small interferring RNA; siYAP, siRNA against YAP; Bax, BCL2 associated X apoptosis regulator.

Downregulation of YAP inhibits the invasiveness of HCC cells in vitro. To investigate whether YAP affects the invasive capabilities of HCC cells, Matrigel invasion assays were performed. It was demonstrated that downregulation of YAP in SMMC-7721 cells significantly decreased cell invasion rates by $41.3 \%$ compared with control cells (Fig. 3). Furthermore, overexpression of YAP in the knockdown cells rescued cell invasion. These results indicate that YAP also serves an important role in the invasiveness of HCC cells. 
Downregulation of YAP inhibits activation of the PI3K/AKT signaling pathway. It has been well documented that the PI3K/AKT pathway is essential for the proliferation and invasion of HCC cells $(18,19)$, and activation of the PI3K/AKT signaling pathway is regulated by YAP $(20,21)$. In the present study, the level of phosphorylated (active) forms of AKT was detected in HCC cells transfected with YAP siRNA. As shown in Fig. 4A, YAP depletion significantly decreased AKT activation, but not the expression of AKT in SMMC-7721 cells. These results suggest that YAP serves a role in the activation of PI3K/AKT pathway in HCC cells.

Downregulation of YAP enhances the sensitivity of HCC cells to CDDP through modulating the PI3K/AKT signaling pathway. Accumulating evidence has indicated that YAP is involved in drug resistance in a variety of cancer cells (22-24). The present study examined whether YAP is involved in drug resistance in HCC cells. To investigate the effect of YAP silencing on the sensitivity of HCC cells to a common clinical chemotherapeutic medicine, CDDP, siYAPor control siRNA-transfected HCC cells were exposed to different concentrations of CDDP ranging between 0 and $80 \mu \mathrm{M}$ for $48 \mathrm{~h}$. As shown in Fig. 4B, the cell survival rate was reduced in a dose-dependent in response to CDDP treatment in all three groups; however, the knockdown of YAP significantly enhanced the sensitivity of SMMC-7721 cells to CDDP treatment. CDDP suppressed the phosphorylation of AKT in SMMC-7721 cells. However, knockdown of YAP induced a lower reduction of $\mathrm{p}$-AKT expression, while the total AKT was unaltered (Fig. 4C). The MTT assay results revealed that LY294002 (a specific PI3K inhibitor) effectively enhanced YAP siRNA-mediated CDDP sensitivity of HCC cells (Fig. 4D). Taken together, these results suggest that knockdown of YAP enhanced the sensitivity of HCC cells to CDDP by suppression of AKT activity.

Downregulation of YAP inhibits tumorigenic growth in the PDTX model. A liver PDTX model was used to investigate the function of YAP in vivo. $\mathrm{Nu} / \mathrm{Nu}$ mice were implanted with different types of patient HCC tumors and assigned to the following four groups: injection with stroke-physiology saline solution (NS), cisplatin (CDDP), YAP-shRNA lentivector (sh-YAP) and CDDP in combination with YAP-shRNA lentivector (CDDP+sh-YAP). As shown in Fig. 5, the tumor volume and weight of mice with sh-YAP was smaller compared with the NS group, suggesting that YAP knockdown significantly suppressed tumorigenic growth in vivo. Furthermore, the tumor volume and weight were significantly smaller in the CDDP+sh-YAP group compared with the CDDP group, suggesting that YAP knockdown in combination with CDDP exhibited more effective anti-tumor properties compared with CDDP alone.

\section{Discussion}

YAP has been proven to be upregulated in various types of cancer, including HCC $(11,25)$. In the present study, it was revealed that overexpression of YAP in HCC is associated with the tumor differentiation. This result is consistent
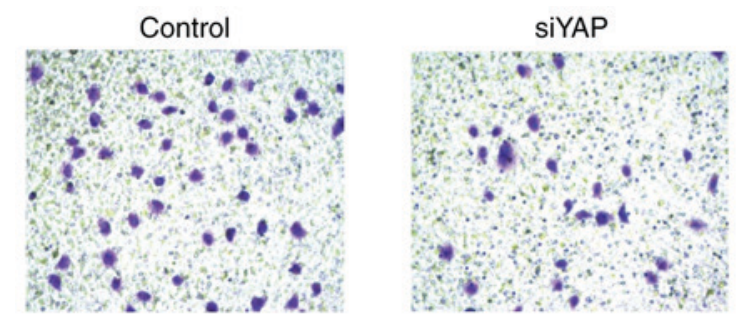

Rescue
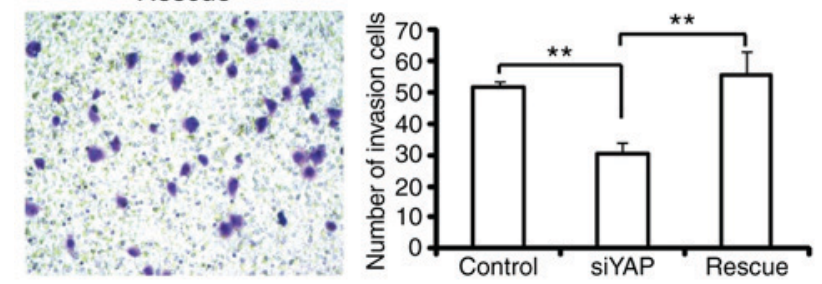

Figure 3. YAP knockdown inhibits hepatocellular carcinoma cell invasion. SMMC-7721 cells transfected with YAP siRNA or rescued with YAP cDNA as indicated were subjected to a Transwell invasion assay. The number of cells invading through the Matrigel are presented. Magnification, x100. ${ }^{* *} \mathrm{P}<0.01$. YAP, Yes-associated protein; CDDP, cisplatin; siRNA/si, small interferring RNA; siYAP, siRNA against YAP.

with a previous study which reported that YAP inhibition restores hepatocyte differentiation in advanced HCC, leading to tumor regression (26). YAP is reported to be involved in several important processes, including cell proliferation, epithelial-to-mesenchymal transition, invasion and metastasis $(7,15,27)$. However, the actual biological functions of YAP and the underlying mechanisms in HCC have not yet been well described. The present study was designed to address this issue using SMMC-7721, a HCC cell line that expresses high levels of YAP.

In the present study, it was demonstrated that siRNA targeting of YAP in SMMC-7721 cells led to the efficient and specific inhibition of endogenous YAP mRNA and protein in vitro. It was revealed that downregulation of YAP in SMMC-7721 cells significantly reduced the invasive capacity of cells. This suggests that YAP is associated with the metastatic events of HCC cells. These data are consistent with the previous findings on other cancer cells, including gastric and lung cancer cells $(7,28)$. Further experiments demonstrated that knockdown of YAP in SMMC-7721 cells significantly inhibited cell proliferation in vitro. In addition, flow cytometry analysis showed that transfection of YAP siRNA induced apoptosis of SMMC-7721 cells. In accordance with these results, transfection of YAP siRNA resulted in the elevation of caspase 3, Bax and p21, as well as reduced c-myc expression in SMMC-7721 cells. Taken together, these results indicated that YAP serves an important role in regulating the proliferation and invasion of HCC cells.

Previous studies revealed that YAP is involved in the activation of numerous signaling pathways, including mitogen activated protein kinase (MAPK)-extracellular signal-regulated kinase signaling pathway (20). However, its involvement in the activation of the PI3K/AKT signaling pathway in HCC has not yet been reported. In the current study, it was observed that YAP silencing triggered the inactivation of AKT in HCC cells, suggesting that the activation of PI3K/AKT is regulated by YAP in HCC cells. It is well known that the activated 
A
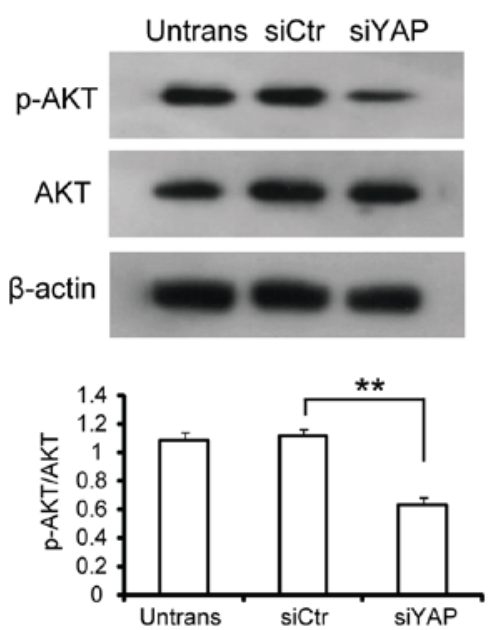

B

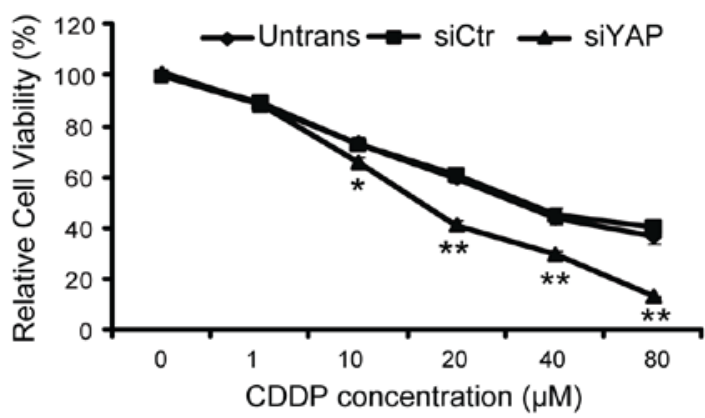

C

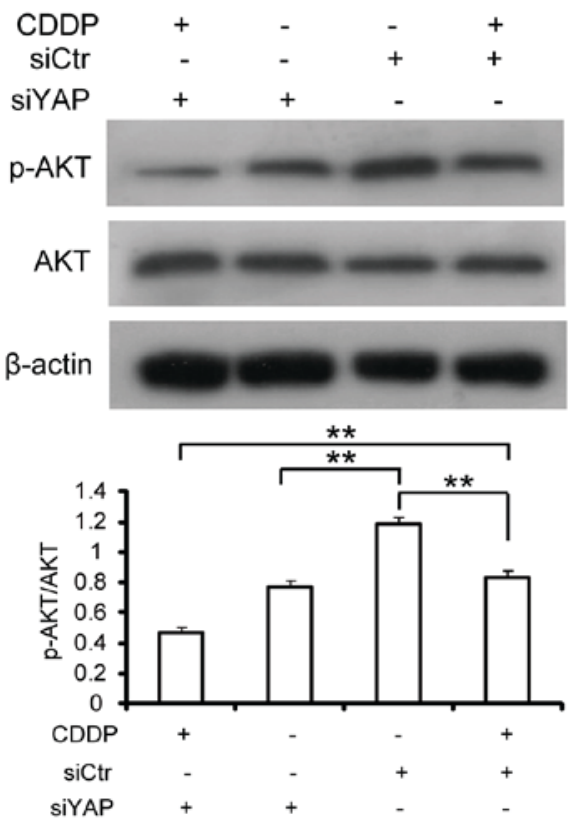

D

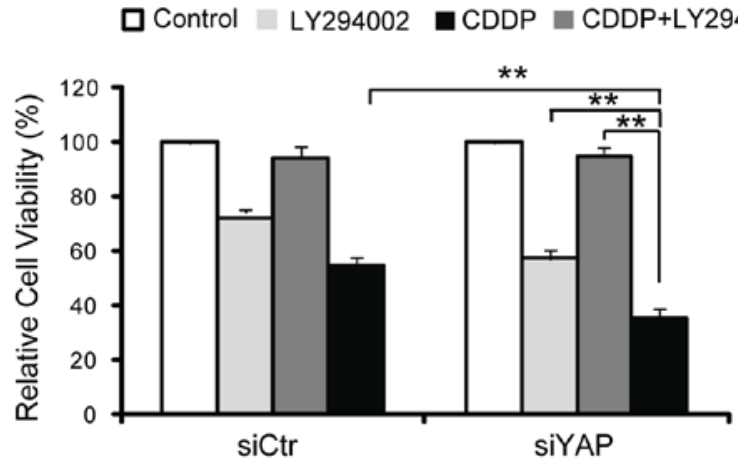

Figure 4. Knockdown of YAP enhances sensitivity of hepatocellular carcinoma cells to CDDP through modulating phosphoinositide 3-kinase/AKT signaling. (A) SMMC-7721 cells were transfected with control siRNA or siRNA against YAP. Then, $48 \mathrm{~h}$ later proteins were extracted and subjected to western blot analysis using antibodies against AKT and p-AKT. (B) Untransfected and siRNA-transfected cells were seeded at 4x10 3 cells/well in 96-well plates and treated with different concentrations of CDPP for $48 \mathrm{~h}$. Then, cell viability was determined using an MTT assay. (C) Western blot analysis of AKT and p-AKT in SMMC-7721 cells transfected with control siRNA or siYAP following treatment with CDDP for $48 \mathrm{~h}$. (D) SMMC-7721 cells transfected with control siRNA or siYAP were treated with $10 \mu \mathrm{M}$ LY294002 for $12 \mathrm{~h}$ prior to being treated with CDDP. Then, cell viability was determined using an MTT assay. ${ }^{\mathrm{P}}<0.05$; ${ }^{* *} \mathrm{P}<0.01$. YAP, Yes-associated protein; CDDP, cisplatin; siRNA/si, small interferring RNA; AKT, AKT serine/threonine kinase; p-, phosphorylated; siCtr, control siRNA; siYAP, siRNA against YAP; Untrans, untransfected.

PI3K/AKT signaling pathway directly modulates the growth, migration and invasion of numerous types of cancer cells, including HCC cells $(18,19)$. Therefore, it is reasonable to hypothesize that the decreased cell proliferation and invasion rates observed in YAP siRNA-transfected HCC cells were due to decreased PI3K/AKT activity.

A number of recent studies have demonstrated that the increased level of YAP was revealed to be associated with increased drug resistance in multiple lines of cancer cells $(23,24,29)$. In the present study, YAP silencing sensitized HCC cells to CDDP, suggesting that YAP induces CDDP resistance in HCC cells. Constitutive activation of the PI3K/AKT pathway has been considered to confer cancer cell resistance to numerous chemotherapy agents, including CDDP (30,31). Thus, in the present study, the PI3K/AKT signaling pathway was investigated to determine whether it served a role in YAP-mediated CDDP resistance in HCC cells. The results revealed that LY294002, an inhibitor of PI3K/AKT, effectively enhanced YAP siRNA-mediated CDDP sensitivity. These findings indicated that YAP regulates chemosensitivity to CDDP through the PI3K/AKT signaling pathway. However, a study by Huo et al (32) suggested that overexpression of YAP in HCC cells conferred doxorubicin resistance through activation of the MAPK signaling pathway, but not the PI3K/AKT pathway. The discrepancy between the two studies may be due to different HCC cell lines and chemotherapeutic agents used. YAP interacting with distinct substrates, or binding partners following treatments with different chemotherapeutic agents, may also be able to explain this discrepancy. The function of YAP was further investigated in vivo using the PDTX model. The data from the current study demonstrated that 

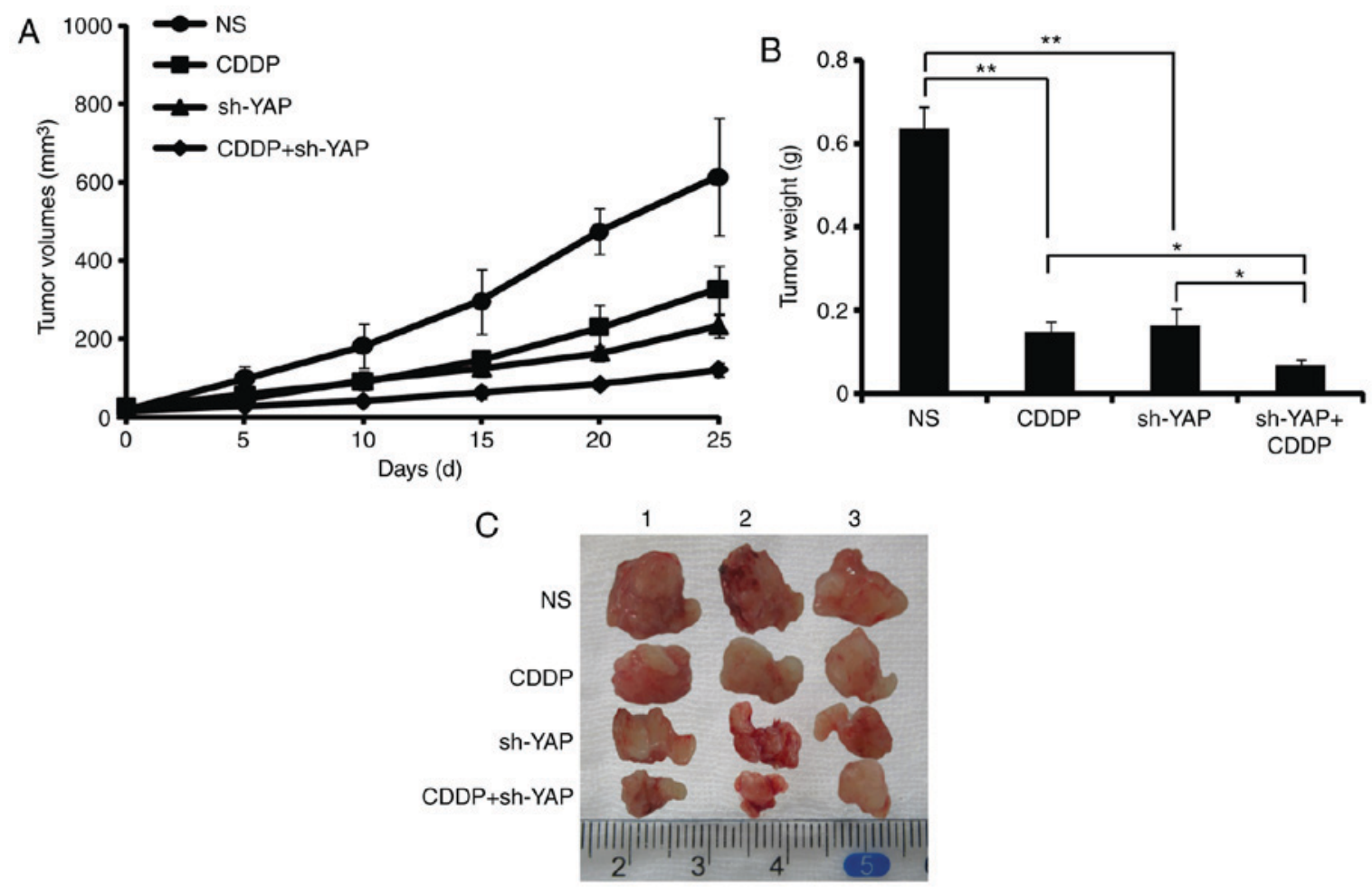

Figure 5. Inhibitory effect of YAP knockdown on in vivo tumor growth in a PDTX model. Patient-derived hepatocellular carcinoma cells were subcutaneously established in BALB/c nude mice. When the tumors reached $100-200 \mathrm{~mm}^{3}$ in size, mice were injected with stroke-physiology saline solution (NS), cisplatin (CDDP), YAP-shRNA lentivector (sh-YAP) and CDDP in combination with YAP-shRNA lentivector (CDDP+sh-YAP) for 25 days. (A and B) The growth curves and the average weights of tumors from nude mice. (C) Representative images of dissected tumors from nude mice. ${ }^{*} \mathrm{P}<0.05,{ }^{* *} \mathrm{P}<0.01 \mathrm{vs}$. NS; ${ }^{*} \mathrm{P}<0.05 \mathrm{vs}$ sh-YAP. NS, stroke-physiology saline solution; CDDP, cisplatin; sh-YAP, YAP-shRNA lentivector; CDDP+sh-YAP, cisplatin in combination with YAP-shRNA lentivector; shRNA/sh, short hairpin RNA; YAP, Yes-associated protein; PDTX, patient-derived tumor xenograft.

YAP knockdown was capable of suppressing tumor growth, increasing the anti-tumor effects of CDDP in the PDTX model. These results indicate that YAP is required for the growth of HCC, and that YAP knockdown in combination with CDDP exhibits more effective anti-tumor properties compared with CDDP alone.

In conclusion, the present study demonstrated that overexpression of YAP in HCC is associated with the tumor differentiation. Knockdown of YAP by siRNA in vitro inhibited the proliferation and invasion of HCC cells, and increased the sensitivity of HCC cells to CDDP, partially by inactivating the PI3K/AKT signaling pathway. In vivo studies using PDTX model suggested a promotive role for YAP in the growth of $\mathrm{HCC}$, and that the knockdown of YAP increases the anti-tumor activity of CDDP. Therefore, these findings suggest that the inhibition of YAP, alone or in combination with traditional chemotherapy, may be considered as an effective anti-HCC strategy.

\section{Acknowledgements}

Not applicable.

\section{Funding}

The present study was supported by a project grant from the Technology Department of Jiaxing (grant no. 2014AY21031-1).

\section{Availability of data and material}

All data generated or analyzed during this study are included in this published article.

\section{Authors' contributions}

XGW was a major contributor in writing the manuscript. $\mathrm{XGW}$ and ZXZ collected and analyzed the experimental data. XGW and BW performed the histological examination of HCC tissues and performed the experiments of $\mathrm{HCC}$ cells in vivo and in vitro. $\mathrm{ZXZ}$ supervised the work of the research group. All authors read and approved the final manuscript.

\section{Ethics approval and consent to participate}

This study was approved and supervised by the Research Ethics Committee of Jiaxing University College of Medicine (Jiaxing, China). Written informed consent was obtained from all patients.

\section{Consent for publication}

The patient, or parent, guardian or next of kin (in case of deceased patients) provided written informed consent for the publication of any associated data and accompanying images. 


\section{Competing interests}

The authors declare that they have no competing interests.

\section{References}

1. Ghouri YA, Mian I and Rowe JH: Review of hepatocellular carcinoma: Epidemiology, etiology, and carcinogenesis. J Carcinog 16: 1, 2017.

2. Burkhart RA, Ronnekleiv-Kelly SM and Pawlik TM: Personalized therapy in hepatocellular carcinoma: Molecular markers of prognosis and therapeutic response. Surg Oncol 26 138-145, 2017.

3. Mazzoccoli G, Miele L, Oben J, Grieco A and Vinciguerra M Biology, epidemiology, clinical aspects of hepatocellular carcinoma and the role of sorafenib. Curr Drug Targets 17: 783-799, 2016.

4. Zhu YJ, Zheng B, Wang HY and Chen L: New knowledge of the mechanisms of sorafenib resistance in liver cancer. Acta Pharmacol Sin 38: 614-622, 2017.

5. Cai WF, Wang L, Liu GS,Zhu P,PaulC and Wang Y: Manipulating the Hippo-Yap signal cascade in stem cells for heart regeneration. Ann Palliat Med 5: 125-134, 2016.

6. Xiao Y, Leach J, Wang J and Martin JF: Hippo/Yap signaling in cardiac development and regeneration. Curr Treat Options Cardiovasc Med 18: 38, 2016.

7. Zhou Z, Zhu JS and Xu ZP: RNA interference mediated YAP gene silencing inhibits invasion and metastasis of human gastric cancer cell line SGC-7901. Hepatogastroenterology 58: 2156-2161, 2011

8. Zhang J, Wang G, Chu SJ, Zhu JS, Zhang R, Lu WW, Xia LQ, Lu YM, Da W and Sun Q: Loss of large tumor suppressor 1 promotes growth and metastasis of gastric cancer cells through upregulation of the YAP signaling. Oncotarget 7: 16180-16193, 2016.

9. Ahmed AA, Mohamed AD, Gener M, Li W and Taboada E: YAP and the Hippo pathway in pediatric cancer. Mol Cell Oncol 4: e1295127, 2017.

10. Zhang Y, Shen H, Withers HG, Yang N, Denson KE2, Mussell AL, Truskinovsky A, Fan Q, Gelman IH, Frangou C and Zhang J: VGLL4 selectively represses YAP-dependent gene induction and tumorigenic phenotypes in breast cancer. Sci Rep 7: 6190, 2017.

11. Wang L, Shi S, Guo Z, Zhang X, Han S, Yang A, Wen W and Zhu Q: Overexpression of YAP and TAZ is an independent predictor of prognosis in colorectal cancer and related to the proliferation and metastasis of colon cancer cells. PLoS One 8 : e65539, 2013

12. Liu Y, Wang G, Yang Y, Mei Z, Liang Z, Cui A, Wu T, Liu CY and Cui L: Increased TEAD4 expression and nuclear localization in colorectal cancer promote epithelial-mesenchymal transition and metastasis in a YAP-independent manner. Oncogene 35 2789-2800, 2016.

13. Wang C, Zhu ZM, Liu CL, He XJ, Zhang HY and Dong JH: Knockdown of yes-associated protein inhibits proliferation and downregulates large tumor suppressor 1 expression in MHCC97H human hepatocellular carcinoma cells. Mol Med Rep 11: 4101-4108, 2015.

14. Tian Y, Tang B, Wang C, Sun D, Zhang R, Luo N, Han Z, Liang R, Gao Z and Wang L: Metformin mediates resensitivity to 5-fluorouracil in hepatocellular carcinoma via the suppression of YAP. Oncotarget 7: 46230-46241, 2016.

15. Liu S, Miao R, Zhai M, Pang Q, Deng Y, Liu S, Qu K, Liu C and Zhang J: Effects and related mechanisms of serotonin on malignant biological behavior of hepatocellular carcinoma via regulation of Yap. Oncotarget 8: 47412-47424, 2017.
16. Hermanek P and Sobin LH (eds): (International Union Against Cancer), TNM Classification of Malignant Tumors. 4th edition, 2nd rev. Berlin, Germany Springer, 1992.

17. Livak KJ and Schmittgen TD: Analysis of relative gene expression data using real-time quantitative PCR and the 2(-Delta Delta C(T)) method. Methods 25: 402-408, 2001.

18. Hsieh YH, Hsieh SC, Lee CH, Yang SF, Cheng CW, Tang MJ, Lin CL, Lin CL and Chou RH: Targeting EMP3 suppresses proliferation and invasion of hepatocellular carcinoma cells through inactivation of PI3K/Akt pathway. Oncotarget 6: 34859-34874, 2015.

19. Wu L, Zheng J, Chen P, Liu Q and Yuan Y: Small nucleolar RNA ACA11 promotes proliferation, migration and invasion in hepatocellular carcinoma by targeting the PI3K/AKT signaling pathway. Biomed Pharmacother 90: 705-712, 2017.

20. Zhang Y, Yuan J, Zhang X, Yan F, Huang M, Wang T, Zheng X and Zhang M: Angiomotin promotes the malignant potential of colon cancer cells by activating the YAP-ERK/PI3K-AKT signaling pathway. Oncol Rep 36: 3619-3626, 2016.

21. Wang $\mathrm{C}, \mathrm{Gu} \mathrm{C}$, Jeong KJ, Zhang D, Guo W, Lu Y, Ju Z, Panupinthu N, Yang JY, Gagea MM, et al: YAP/TAZ-mediated upregulation of GAB2 leads to increased sensitivity to growth factor-induced activation of the PI3K pathway. Cancer Res 77: 1637-1648, 2017

22. Zhao Y, Khanal P, Savage P, She YM, Cyr TD and Yang X: YAP-induced resistance of cancer cells to antitubulin drugs is modulated by a Hippo-independent pathway. Cancer Res 74: 4493-4503, 2014

23. Keren-Paz A, Emmanuel R and Samuels Y: YAP and the drug resistance highway. Nat Genet 47: 193-194, 2015

24. Hsu PC, You B, Yang YL, Zhang WQ, Wang YC, Xu Z, Dai Y, Liu S, Yang CT, Li H, et al: YAP promotes erlotinib resistance in human non-small cell lung cancer cells. Oncotarget 7: 51922-51933, 2016

25. Li H, Wang S, Wang G, Zhang Z, Wu X, Zhang T, Fu B and Chen $\mathrm{G}$ : Yes-associated protein expression is a predictive marker for recurrence of hepatocellular carcinoma after liver transplantation. Dig Surg 31: 468-478, 2014.

26. Fitamant J, Kottakis F, Benhamouche S, Tian HS, Chuvin N, Parachoniak CA, Nagle JM, Perera RM, Lapouge M, Deshpande V et al: YAP inhibition restores hepatocyte differentiation in advanced HCC, leading to tumor regression. Cell Rep, Mar 10, 2015 (Epub ahead of print)

27. Wang S, Li H, Wang G, Zhang T, Fu B, Ma M, Quan Z and Chen G: Yes-associated protein (YAP) expression is involved in epithelial-mesenchymal transition in hepatocellular carcinoma. Clin Transl Oncol 18: 172-177, 2016.

28. Dong Q, Fu L, Zhao Y, Du Y, Li Q, Qiu X and Wang E: Rab1la promotes proliferation and invasion through regulation of YAP in non-small cell lung cancer. Oncotarget 8: 27800-27811, 2017.

29. Lee JE, Park HS, Lee D, Yoo G, Kim T, Jeon H, Yeo MK, Lee CS, Moon JY, Jung SS, et al: Hippo pathway effector YAP inhibition restores the sensitivity of EGFR-TKI in lung adenocarcinoma having primary or acquired EGFR-TKI resistance. Biochem Biophys Res Commun 474: 154-160, 2016.

30. Fu X, Feng J, Zeng D, Ding Y, Yu C and Yang B: PAK4 confers cisplatin resistance in gastric cancer cells via PI3K/Akt- and MEK/ERK-dependent pathways. Biosci Rep 34: pii: e00094, 2014.

31. Xiong Z, Fu Z, Shi J, Jiang X and Wan H: HtrA1 down-regulation induces cisplatin resistance in colon cancer by increasing XIAP and activating PI3K/Akt pathway. Ann Clin Lab Sci 47: 264-270, 2017.

32. Huo X, Zhang Q, Liu AM, Tang C, Gong Y, Bian J, Luk JM, Xu Z and Chen J: Overexpression of Yes-associated protein confers doxorubicin resistance in hepatocellullar carcinoma. Oncol Rep 29: 840-846, 2013. 